\title{
Perfil da sexualidade de pessoas idosas portadoras de SIDA/AIDS atendidas em um serviço de saúde do Distrito Federal*
}

\author{
Sexuality profile of older people with HIV/ \\ AIDS cared for in a health service of the \\ Federal District
}

Pollyanna Braga Santos de Andrade ${ }^{1}$ Linconl Agudo Oliveira Benito ${ }^{2}$
Recebido em: 10/12/2015.

Aprovado em: 31/10/2016.

1 Graduanda em Enfermagem do Centro Universitário de Brasília (UniCEUB).

2 Docente do Curso de Enfermagem do Centro Universitário de Brasília (UniCEUB).

\section{Resumo}

Estudo exploratório, descritivo e de abordagem quantitativa que analisou a sexualidade de pessoas idosas portadoras de HIV/AIDS. Para aquisição dos dados, foi utilizado enquanto instrumento e coleta de dados (ICD) a Escala de Atitudes e Conhecimento sobre Sexualidade no Envelhecimento (ASKAS). Foram entrevistados dez (10) idosos. Os sujeitos da pesquisa se constituíram de $70 \%(\mathrm{n}=7)$ pessoas com 60 a 69 anos, 70\% $(\mathrm{n}=7)$ do sexo masculino, 50\% $(\mathrm{n}=5)$ nordestinos, $40 \%$ $(n=4)$ se encontravam separados ou divorciados, $40 \%(n=4)$ possuíam ensino médio completo, $70 \%(\mathrm{n}=7)$ eram tabagistas, nenhum etilista, nenhum dependente químico, $40 \%(\mathrm{n}=4)$ possuíam HA, $40 \%$ possuíam DM, 20\% $(n=2)$ possuíam outra enfermidade, $60 \%(n=6)$ possui HIV/ AIDS a menos de 10 anos, $50 \%(n=5)$ consomem pelo menos cinco (5) ou mais medicações, $80 \%(\mathrm{n}=8)$ possuíam renda mensal de 3 ou mais salários mínimos, $40 \%(\mathrm{n}=4)$ moram numa residência com mais de 3 pessoas, e $70 \%(n=7)$ declararam possuir filhos. Foi possível evidenciar que as pessoas idosas portadoras de HIV/AIDS possuem conhecimento em relação a sexualidade, sendo esta um fator relacionado a qualidade de vida (QV). Paradoxalmente aos avanços tecnológicos e a inovações científicas, ainda são percebidos por meio dos discursos, preconceitos em relação à sexualidade e medo de não serem capazes de desenvolverem a sexualidade.

Palavras-chave: Idoso. Sexualidade. HIV. Síndrome da Imunodeficiência Adquirida.

\footnotetext{
Abstract

A scope and descriptive study with a quantitative approach analyze sexuality among HIV-positive senior citizens. For this research data acquisition was used, for Data Collection Tool (ICD- monogram in Portuguese), the Aging Sexual Attitude and Knowledge Scale (ASKAS). This survey was conducted on 10 senior citizens. The subjects for this research were $70 \%$ (7) between 60 and 69 years old, 70\% (7) were male, $50 \%$ were northeastern, $40 \%$ (4) were divorced or separated, $40 \%$ (4) had concluded high school, 70\% (7) were smokers, non were alcoholic, non were chemical dependent, 40\% (4) had Diabetes Mellitus (DM), 40\% had Arterial Hypertension (AH), 20\% (2) had another illness, 60\% (6) were HIV-positive for less than 10 years, 50\% were taking five (5) or more
} 
medications, $80 \%$ had an income of 3 times the monthly minimum wage, 40\% (4) were living with more than 3 people, and $70 \%$ (7) declared that they had children. It was possible to show that HIV-positive senior citizens were knowledgeable about sexuality, which is a factor related to life quality (LQ). Paradoxically, the advance of technology and scientific innovations are yet noticed through discussions, prejudice related to the topic sexuality, and the fear of developing sexual capability.

Keywords: Senior Citizens. Sexuality. HIV. Acquired Immunodeficiency Syndrome.

\section{Introdução}

Em decorrência do aumento da longevidade e das opções que a vida moderna dispõe, como por exemplo a reposição hormonal e as medicações para impotência sexual, a população idosa vem redescobrindo experiências, uma delas é o sexo. Entretanto, as práticas sexuais inseguras, fazem com que os indivíduos acima de 60 anos estejam mais vulneráveis a contaminar-se pelo Vírus da Imunodeficiência Humana (HIV), agente causador da Síndrome da Imunodeficiência Adquirida (SIDA/AIDS) (LAROQUE et al., 2011).

De acordo com Cruz e Ramos (2012), a saúde dos idosos está relacionada com a funcionalidade global, que expressa à capacidade do indivíduo administrar e cuidar da própria vida. O idoso é considerado ativo quando é capaz de ser independente e autônomo para realizar as atividades do seu dia a dia, mesmo sendo portador de alguma patologia. Devido à natureza complexa dos problemas multidimensionais que afetam a capacidade funcional da população de idade mais avançada, destaca-se a infecção pelo HIV/AIDS, visto que essa doença possui um quadro clínico limitante mesmo por meio de manifestações simples, sendo considerada do grupo de doenças crônicas graves que pode comprometer o desempenho funcional dos idosos e afetar significativamente a qualidade de vida dessa população.

Atualmente, encontramos indivíduos acima de 50 anos que consideram improvável eles adquirirem alguma infecção sexualmente transmissível (IST). Essa ideia pode estar associada à prerrogativa de que o sexo acontece na juventude e isso pode contribuir para manter esse grupo excluído das ações preventivas. Tanto os idosos como os profissionais de saúde têm dificuldades para compreender e manifestar suas opiniões sobre sexualidade nessa fase da vida. A invisibilidade da sexualidade pode implicar uma assistência fragmentada, visto que muitas ações como a prevenção de IST não são zada com médicos, verificou-se que, dificilmente, esses profissionais indagam os idosos sobre sua vida sexual e, consequentemente, deixam de preveni-los quanto a AIDS, o que pode resultar em um diagnóstico tardio e em um aumento da exposição ao vírus (MACHIESQUI et al., 2010).

Diante disso, faz-se necessário o estabelecimento de políticas públicas e estratégias de prevenção às IST/ AIDS que possam garantir o bem-estar dos indivíduos idosos, pois, devido ao aumento dessa patologia nesse grupo etário, sua prevenção se torna um desafio para o Brasil. Além disso, questões sobre a AIDS no envelhecimento requer maior aprofundamento em discussões sobre o tema, com o objetivo de proporcionar subsídios relacionados aos cuidados com os portadores do HIV/ AIDS e ao desenvolvimento de ações dos programas de prevenção (GIRONDI et al., 2012).

Para o Instituto Brasileiro de Geografia e Estatística (IBGE), idoso é o indivíduo acima de 60 anos. A Organização Mundial de Saúde (OMS) considera o mesmo limite de idade para os países em desenvolvimento. Conforme a literatura, houve um aumento no número de idosos no Brasil, somando 11, 1\% da população com um total de 21 milhões de pessoas. Constatou-se, por meio de projeções, que, em 2050, a população idosa será em torno de $20 \%$ da população total brasileira. Por causa do avanço no campo da saúde e da queda nas taxas de fecundidade e mortalidade, houve um aumento da expectativa de vida da população brasileira, ocasionando diminuição da população jovem e aumento na mesma proporção do número de idosos (BASTOS et al., 2012).

Por causa do caráter pandêmico da AIDS, ela é considerada uma doença que representa um dos maiores problemas de saúde da atualidade. Os infectados pelo HIV evoluem para uma grave disfunção do sistema imunológico na medida em que os linfócitos T CD4+ vão sendo destruídos, pois é uma das principais células-alvo do vírus. A contagem de linfócitos T CD4+ é um importante marcador dessa imunodeficiência, sendo 
utilizado para estimar o prognóstico, avaliar indicação para inicio da terapia antirretroviral e definir os casos de AIDS. A história natural dessa infecção vem sendo alterada pela terapia antirretroviral, que foi iniciada no Brasil em 1996 como resultado no aumento da sobrevida dos pacientes mediante a reconstrução das funções do sistema imunológico e da redução das doenças secundárias. A transmissão do vírus pode ser por via sexual (esperma e secreção vaginal), pelo sangue (via parenteral e vertical) e pelo leite materno. O portador do HIV é transmissor desde o momento que a infecção for adquirida. Os indivíduos com infecção aguda têm maior concentração do HIV no sangue e nas secreções sexuais, tendo uma maior facilidade para transmitir o vírus (BRASIL, 2010).

O preconceito e a dificuldade que se têm para criar medidas de prevenção, principalmente no que se concerne à utilização de preservativos no público idoso, é mais intenso do que nos outros segmentos populacionais, sendo uma das razões de poucas campanhas para esse público. A possibilidade de o idoso ser infectado pelo HIV é imperceptível à sociedade e a essa população, uma vez que não estão acostumados a utilizar o preservativo. Além disso, as mulheres idosas que geralmente não podem engravidar têm a falsa impressão da inútil utilização do preservativo. Com isso, há uma falta de identificação desse público com as campanhas de prevenção da AIDS, que tem sempre como foco a população jovem, contribuindo para que o idoso não se considere um doente em potencial (MASCHIO et al., 2011).

Portanto, o objetivo deste estudo foi analisar a sexualidade de pessoas idosas portadoras de SIDA/AIDS atendidas em departamento especializado.

\section{Metodologia}

O presente estudo utilizou-se de uma pesquisa de campo, exploratória, e de natureza quantitativa, que analisou a questão da sexualidade de pessoas idosas portadoras de SIDA/AIDS. A pesquisa foi iniciada após a autorização expressa do Comitê de Ética e Pesquisa (CEP) da Fundação de Ensino e Pesquisa em Ciências da Saúde (FEPECS) da Secretaria de Estado de Saúde do Distrito Federal (SES-DF) pelo parecer de nº 994.842 emitido no dia 23 de março de 2015, respeitando a resolução que regulamenta a pesquisa com seres humanos no Brasil, resolução n 466/2012 do Conselho Nacional de Saúde (CNS. A pesquisa teve início somente após a assinatura do Termo de Consentimento Livre e Esclarecido (TCLE) pelos participantes.

Para a aquisição dos dados, optou-se pela utilização de um instrumento de coleta de dados possuidor de três (03) partes. A primeira parte se refere ao Mini-Exame do Estado Mental (MEEM), questionário que ajudou a identificar se o (a) idoso (a) possuía condições de participar da pesquisa. Já na segunda parte do ICD, foi aplicado um questionário contendo um quantitativo de dezoito (18) perguntas, que permitiu a construção do perfil socioeconômico dos idosos. Na terceira parte do ICD, foi utilizada a Escala de Atitudes e Conhecimento sobre Sexualidade no Envelhecimento (ASKAS), adaptada por Viana, Guirardello e Madruga (2010), que permitiu a análise da sexualidade dos idosos que participaram da pesquisa, sendo empregada somente a primeira parte da escala, pois a parte II está direcionada para idosos institucionalizados, que não é o caso dos participantes deste estudo.

Os critérios estabelecidos para aplicação do questionário se constituem de pessoas com faixa etária superior a 60 anos de idade, de ambos os sexos, que fossem portadores de SIDA/AIDS, que sejam regularmente atendidos e cadastrados no Programa de DST juntamente a uma instituição pública de saúde administrada pela Secretaria de Estado de Saúde do Distrito Federal (SES-DF), e que possuíssem condições psicológicas e interesse em participar desta pesquisa.

As fontes secundárias se constituíram de artigos de periódicos científicos juntamente às bases de dados Medline, Lilacs, Scielo, Bireme, utilizando enquanto descritores as palavras "idoso", "sexualidade", "SIDA/ AIDS". O recorte histórico se constituiu dos anos de 2005 a 2014.

Os dados pessoais dos entrevistados foram transformados em códigos numéricos e as respostas dos idosos às escalas foram submetidas a análises estatísticas exploratórias, descritivas, como: percentagem. A execução das análises estatísticas foi organizada por meio do software Microsoft Excel 2013 for Windows ${ }^{\circledR}$.

A pesquisa foi composta por pessoas com faixa etária superior a 60 anos de idade, do sexo feminino e masculino portadores de SIDA/AIDS e que estão cadastrados no Programa de DST junto à Policlínica de Taguatinga da Secretaria de Estado de Saúde do Distrito Federal (SES-DF) e que se manifestaram interessados 
e em condições psicológicas para participar desta pesquisa. Dentre os pacientes cadastrados no Programa de DST da Unidade Mista de Taguatinga, 24 não entram nos critérios estabelecidos da pesquisa.

A referida descrição foi composta por um quantitativo de 10 pessoas idosas com diagnóstico positivado de SIDA/AIDS, e que recebem tratamento junto à Policlínica de Taguatinga, integrante do Hospital Regional de Taguatinga (HRT) da Secretaria de Estado de Saúde do Distrito Federal (SES-DF).

\section{Resultados}

Conforme registro na Unidade Mista de Taguatinga, verificamos que existem 46 pacientes acima de 60 anos cadastrados no Programa de DST com o diagnóstico positivado de AIDS, deste universo: 52,15\% $(\mathrm{n}=24)$ não entram nos parâmetros da pesquisa, pois 08 estão em abandono de acompanhamento terapêutico, 09 diagnosticados com o quadro de demência, 01 falecido, 01 sem condições emocionais para participar da pesquisa pelo falecimento do cônjuge, 03 faltaram à entrevista e 02 se negaram a participar da pesquisa; $26,1 \%(n=12)$ estão com os dados para contatos desatualizados, pois foram realizadas várias tentativas de contato sem sucesso, dando os números telefônicos como "inexistente", "errado" ou "cortado"; $21,75 \%(\mathrm{n}=10)$ se disponibilizaram a realizar a entrevista.

Em relação ao perfil socioeconômico dos entrevistados $(n=10)$, as maiores proporções obtidas foram: $70 \%(n=7)$ com idade entre 60 a 69 anos, $70 \%$ $(\mathrm{n}=7)$ do sexo masculino, $50 \%(\mathrm{n}=5)$ natural do Nordeste, $40 \%(n=4)$ com estado civil separado ou divorciado, 40\% $(\mathrm{n}=4)$ com nível médio completo, 100\% $(\mathrm{n}=10)$ não são etilistas, $70 \%(\mathrm{n}=7)$ não são tabagistas, $100 \%(n=10)$ não são dependentes químicos, $60 \%$ $(n=6)$ não são portadores de hipertensão arterial, $60 \%(n=6)$ não são portadores de diabetes mellitus, $80 \%(n=8)$ não possuem outras enfermidades, $60 \%$ $(n=6)$ têm o tempo de diagnóstico com SIDA/AIDS menor que 10 anos, 50\% $(n=5)$ consomem acima de três medicações diárias, $80 \%(\mathrm{n}=8)$ possuem renda mensal de 03 ou mais salários mínimos, $40 \%(n=4)$ residem com mais de três pessoas, $70 \%(n=7)$ possuem filhos (Tabela 1 ).
Tabela 1 - Perfil socioeconômico dos sujeitos da pesquisa $(n=10)$

\begin{tabular}{|c|c|c|}
\hline Idade & Frequência & $\%$ \\
\hline 60 a 69 anos & 7 & 70 \\
\hline 70 a 79 anos & 3 & 30 \\
\hline \multicolumn{3}{|l|}{ Sexo } \\
\hline Masculino & 7 & 70 \\
\hline Feminino & 3 & 30 \\
\hline \multicolumn{3}{|l|}{ Naturalidade } \\
\hline Nordeste & 5 & 50 \\
\hline Sudeste & 4 & 40 \\
\hline Centro-oeste & 1 & 10 \\
\hline \multicolumn{3}{|l|}{ Estado civil } \\
\hline Separado/Divorciado (a) & 4 & 40 \\
\hline Solteiro & 3 & 30 \\
\hline Casado (a) & 2 & 20 \\
\hline Outros & 1 & 10 \\
\hline \multicolumn{3}{|l|}{ Escolaridade } \\
\hline Nível Médio & 4 & 40 \\
\hline Nível Fundamental & 3 & 30 \\
\hline Nível Superior & 3 & 30 \\
\hline Outros & 0 & 0 \\
\hline \multicolumn{3}{|l|}{ Etilista } \\
\hline Não & 10 & 100 \\
\hline \multicolumn{3}{|l|}{ Tabagista } \\
\hline Não & 7 & 70 \\
\hline Sim & 3 & 30 \\
\hline \multicolumn{3}{|l|}{ Dependente químico } \\
\hline Não & 10 & 100 \\
\hline \multicolumn{3}{|c|}{ Portador de hipertensão arterial } \\
\hline Não & 6 & 60 \\
\hline Sim & 4 & 40 \\
\hline \multicolumn{3}{|l|}{ Portador de diabetes mellitus } \\
\hline Não & 6 & 60 \\
\hline Sim & 4 & 40 \\
\hline \multicolumn{3}{|l|}{ Outra enfermidade } \\
\hline Não & 8 & 80 \\
\hline Sim & 2 & 20 \\
\hline \multicolumn{3}{|c|}{ Tempo diagnosticado com SIDA/AIDS } \\
\hline$<10$ anos & 6 & 60 \\
\hline$>10$ anos & 4 & 40 \\
\hline \multicolumn{3}{|c|}{ Consumo de medicações diárias } \\
\hline 2 & 3 & 30 \\
\hline 1 & 1 & 10 \\
\hline 3 & 1 & 10 \\
\hline Acima de três & 5 & 50 \\
\hline \multicolumn{3}{|l|}{ Renda mensal } \\
\hline 3 ou mais salários mínimos & 8 & 80 \\
\hline 1 salário mínimo & 1 & 10 \\
\hline 2 salários mínimos & 1 & 10 \\
\hline \multicolumn{3}{|c|}{ Pessoas moradoras na residência } \\
\hline 1 & 3 & 30 \\
\hline 2 & 2 & 20 \\
\hline 3 & 1 & 10 \\
\hline Acima de três & 4 & 40 \\
\hline \multicolumn{3}{|l|}{ Possui filho(s) } \\
\hline Sim & 7 & 70 \\
\hline Não & 3 & 30 \\
\hline Total & 10 & 100 \\
\hline
\end{tabular}

Fonte: Produção dos autores 
Em relação à sexualidade dos idosos entrevistados, utilizamos à Escala de Atitudes e Conhecimento sobre Sexualidade no Envelhecimento (ASKAS), em que os sujeitos da pesquisa tinham três opções para responder cada pergunta: verdadeiro, falso ou não sei. No primeiro questionamento, perguntamos se a Atividade sexual em pessoas idosas é frequentemente perigosa para sua saúde, 70\% (n= 7) dos entrevistados responderam que é falso. Homens acima de 65 anos normalmente levam mais tempo para conseguir uma ereção do pênis do que os homens mais jovens, $90 \%(n=9)$ responderam verdadeiro. A sexualidade é, geralmente, uma necessidade que se faz presente durante a vida toda, $90 \%(n=9)$ responderam verdadeiro. Há evidencias de que a atividade sexual em pessoas com mais de 65 anos traz benefícios físicos, 70\% $(n=7)$ responderam verdadeiro. A atividade sexual pode trazer benefícios psicológicos para a pessoa com mais de 65 anos, 100\% ( $\mathrm{n}=$ 10) responderam verdadeiro. A maioria das mulheres com mais de 65 anos é fria sexualmente, 50\% $(n=5)$ responderam verdadeiro. Medicamentos podem alterar o desejo sexual de uma pessoa, 70\% $(n=7)$ responderam verdadeiro. Em geral, as mudanças na sexualidade das pessoas com mais de 65 anos, tem mais relação com respostas mais lentas do que com diminuição do interesse por sexo, 90\% $(n=9)$ responderam verdadeiro. Mulheres e homens com mais de 65 anos não podem ser parceiros sexuais entre si, pois tanto um quanto o outro precisam de parceiros mais jovens para ser estimulados, $80 \%(n=8)$ responderam verdadeiro. Tranquilizantes e álcool podem diminuir os níveis de excitação sexual em pessoas com mais de 65 anos e interferir na resposta sexual, $100 \%(n=10)$ responderam verdadeiro. Com o aumento da idade, há uma queda na frequência das atividades sexuais em homens, 90\% ( $n=9)$ responderam verdadeiro. Um fator importante na manutenção da resposta sexual em homens com mais de 65 anos é a continuidade da atividade sexual ao longo de sua vida, $100 \%(n=10)$ responderam verdadeiro. O medo de não ser capaz de realizar o ato sexual pode acarretar incapacidade no desempenho sexual em homens com mais de 65 anos, $100 \%(n=10)$ responderam verdadeiro. É provável que o término da atividade sexual em pessoas com mais de 65 anos se deva mais a fatores sociais e psicológicos do que a fatores biológicos e físicos, $70 \%(n=7)$ responderam verdadeiro. A masturbação em excesso pode causar o aparecimento de confusão mental e de demência em pessoas com mais de 65 anos, $60 \%(n=6)$ responderam verdadeiro. Nas mulheres, a perda de satisfação sexual é inevitável após a menopausa, 50\% $(n=5)$ responderam falso. A impotência de causa não orgânica aumenta em homens com mais de 65 anos em comparação com homens mais jovens, $50 \%(n=5)$ responderam verdadeiro. Em muitos casos, a impotência em homens com mais de 65 anos pode ser realmente tratada e curada efetivamente, $80 \%(n=8)$ responderam verdadeiro. $\mathrm{Na}$ ausência de problemas físicos graves, mulheres e homens podem manter o interesse e atividades sexuais até depois de 80 ou 90 anos de idade, 100\% $(n=10)$ responderam verdadeiro. A masturbação em homens e mulheres com mais de 65 anos traz benefícios para a manutenção da resposta sexual, $60 \%(n=6)$ responderam verdadeiro (Quadro 2).

Quadro 2 - Escala de Atitudes e Conhecimento sobre Sexualidade no Envelhecimento (ASKAS)

\begin{tabular}{|c|c|c|}
\hline 1. A Atividade sexual em pessoas idosas é frequentemente perigosa para sua saúde. & Frequência & $\%$ \\
\hline Verdadeiro & 3 & 30 \\
\hline Falso & 7 & 70 \\
\hline \multicolumn{3}{|c|}{ 2. Homens com mais de 65 anos normalmente levam mais tempo para conseguir uma ereção do pênis do que os homens mais jovens. } \\
\hline Verdadeiro & 9 & 90 \\
\hline Falso & 1 & 10 \\
\hline \multicolumn{3}{|l|}{ 3. A sexualidade é geralmente uma necessidade que se faz presente durante a vida toda. } \\
\hline Verdadeiro & 9 & 90 \\
\hline Falso & 1 & 10 \\
\hline \multicolumn{3}{|c|}{ 4. Há evidências de que a atividade sexual em pessoas com mais de 65 anos traz benefícios físicos. } \\
\hline Verdadeiro & 7 & 70 \\
\hline Falso & 3 & 30 \\
\hline \multicolumn{3}{|l|}{ 5. A atividade sexual pode trazer benefícios psicológicos para a pessoa com mais de 65 anos. } \\
\hline Verdadeiro & 10 & 100 \\
\hline \multicolumn{3}{|l|}{ 6. A maioria das mulheres com mais de 65 anos é fria sexualmente. } \\
\hline Verdadeiro & 5 & 50 \\
\hline Falso & 5 & 50 \\
\hline \multicolumn{3}{|l|}{ 7. Medicamentos podem alterar o desejo sexual de uma pessoa. } \\
\hline Verdadeiro & 7 & 70 \\
\hline Falso & 2 & 20 \\
\hline
\end{tabular}




\begin{tabular}{|c|c|c|}
\hline Não sei & 1 & 10 \\
\hline \multicolumn{3}{|c|}{ 8. Em geral, as mudanças na sexualidade das pessoas com mais de 65 anos, tem mais relação com respostas mais lentas do que com diminuição do interesse por sexo. } \\
\hline Verdadeiro & 9 & 90 \\
\hline Não sei & 1 & 10 \\
\hline \multicolumn{3}{|c|}{ 9. Mulheres e homens com mais de 65 anos não podem ser parceiros sexuais entre si, pois tanto um quanto o outro precisam de parceiros mais jovens para serem estimulados. } \\
\hline Verdadeiro & 8 & 80 \\
\hline Falso & 2 & 20 \\
\hline \multicolumn{3}{|c|}{ 10. Tranquilizantes e álcool podem diminuir os níveis de excitação sexual em pessoas com mais de 65 anos e interferir na resposta sexual. } \\
\hline Verdadeiro & 10 & 100 \\
\hline \multicolumn{3}{|c|}{ 11. Com 0 aumento da idade, há uma queda na frequência das atividades sexuais em homens. } \\
\hline Verdadeiro & 9 & 90 \\
\hline Não sei & 1 & 10 \\
\hline \multicolumn{3}{|c|}{ 12. Um fator importante na manutenção da resposta sexual em homens com mais de 65 anos é a continuidade da atividade sexual ao longo de sua vida. } \\
\hline Verdadeiro & 10 & 100 \\
\hline \multicolumn{3}{|c|}{ 13. 0 medo de não ser capaz de realizar o ato sexual pode acarretar incapacidade no desempenho sexual em homens com mais de 65 anos. } \\
\hline Verdadeiro & 10 & 100 \\
\hline \multicolumn{3}{|c|}{ 14. É provável que o término da atividade sexual em pessoas com mais de 65 anos se deva mais a fatores sociais e psicológicos do que a fatores biológicos e físicos. } \\
\hline Verdadeiro & 7 & 70 \\
\hline Falso & 2 & 20 \\
\hline Não sei & 1 & 10 \\
\hline \multicolumn{3}{|c|}{ 15. A masturbação em excesso pode causar 0 aparecimento de confusão mental e de demência em pessoas com mais de 65 anos. } \\
\hline Verdadeiro & 6 & 60 \\
\hline Falso & 2 & 20 \\
\hline Não sei & 2 & 20 \\
\hline \multicolumn{3}{|c|}{ 16. Nas mulheres, a perda de satisfação sexual é inevitável após a menopausa. } \\
\hline Verdadeiro & 4 & 40 \\
\hline Falso & 5 & 50 \\
\hline Não sei & 1 & 10 \\
\hline \multicolumn{3}{|c|}{ 17. A impotência de causa não-orgânica aumenta em homens com mais de 65 anos em comparação com homens mais jovens. } \\
\hline Verdadeiro & 5 & 50 \\
\hline Falso & 3 & 30 \\
\hline Não sei & 2 & 20 \\
\hline \multicolumn{3}{|c|}{ 18. Em muitos casos, a impotência em homens com mais de 65 anos pode ser realmente tratada e curada efetivamente. } \\
\hline Verdadeiro & 8 & 80 \\
\hline Falso & 1 & 10 \\
\hline Não sei & 1 & 10 \\
\hline \multicolumn{3}{|c|}{ 19. Na ausência de problemas físicos graves, mulheres e homens podem manter o interesse e atividades sexuais até depois de 80 ou 90 anos de idade. } \\
\hline Verdadeiro & 10 & 100 \\
\hline \multicolumn{3}{|c|}{ 20. A masturbação em homens e mulheres com mais de 65 anos traz benefícios para a manutenção da resposta sexual. } \\
\hline Verdadeiro & 6 & 60 \\
\hline Falso & 3 & 30 \\
\hline Não sei & 1 & 10 \\
\hline Total & 10 & 100 \\
\hline
\end{tabular}

Fonte: Viana, Guirardello e Madruga (2010)

\section{Discussão}

Em análise dos resultados socioeconômico encontrado na Tabela 1, observa-se que 70\% dos sujeitos da pesquisa são do gênero masculino com faixa etária entre 60 e 69 anos (70\%). Porém, houve um crescimento no número de casos de AIDS entre o gênero feminino que, geralmente, têm um poder aquisitivo menor e um maior comprometimento da saúde, causando uma entrega mais rápida a essa doença se comparada aos homens (OKUNO et al., 2012).

Segundo Araújo et al. (2007), no Brasil a via de transmissão heterossexual tem sido um fator importante em relação à dinâmica da epidemia, pois esse atributo tem contribuído para o aumento do número de casos em mulheres. A faixa etária atingida não se restringe somente as jovens, mas observou-se o aumento do número de notificações em idades a partir dos 50 anos. $\mathrm{O}$ crescimento do número de mulheres com AIDS, principalmente heterossexuais, revela a vulnerabilidade feminina e, na maioria dos casos, a dificuldade de negociação do uso do preservativo com o parceiro.

A respeito da sexualidade dos idosos encontrado na Tabela 2, 100\% dos entrevistados acreditam que a 
atividade sexual ao longo da vida é um fator importante para manutenção da resposta sexual, assim como, o interesse e as atividades sexuais devem ser mantidos até depois dos 80/90 anos de idade, expressando o desejo de ter uma vida sexual ativa.

A sexualidade é parte integrante da personalidade do indivíduo. Sua evolução se completa com a satisfação das necessidades humanas básicas, como o prazer, amor, carinho, o desejo de contato, intimidade e expressão emocional (MORAES et al., 2011).

Em relação aos parceiros sexuais, $80 \%$ dos idosos referem preferência por pessoas mais jovens, com a intenção de um maior estimulo na atividade sexual, retratando uma mudança social em relação aos casos amorosos atuais.

É comum que os homens idosos procurem como parceiras sexuais as mulheres mais jovens, no entanto, quando as mulheres idosas se envolvem sexualmente com homens mais novos o olhar da sociedade muda, como se essa atitude fosse insensata ou absurda. $\mathrm{Na}$ verdade, o idoso continua tendo impulso e atividade sexual, mesmo que haja alterações relacionadas às mudanças fisiológicas, expectativas socioculturais, problemas de saúde e medicações (LEITE; MOURA; BERLEZI, 2007).

Os sujeitos da pesquisa (100\%) revelaram que o medo de não ser capaz de realizar o ato sexual pode acarretar incapacidade no desempenho sexual em homens com mais de 65 anos, revelando o sentimento que os idosos internalizaram por meio do discurso que a sociedade expressa quando enxerga no idoso um ser assexuado. Além disso, 70\% dos entrevistados acham que o abandono da atividade sexual em idosos está ligado diretamente aos fatores sociais e psicológicos do que aos fatores biológicos e físicos. Isso revela o sentimento de invisibilidade que o idoso tem em relação à sociedade retratando novamente o preconceito.

De acordo com Almeida e Lourenço (2007), a principal característica da senilidade é o declínio, principalmente físico, que provoca as alterações sociais e psicológicas. Em muitas ocasiões os idosos se excluem das atividades sociais, aceitando que não são mais capazes de manter algum relacionamento ou investir em um novo. $\mathrm{E}$ a sociedade, muitas vezes, contribui para que os idosos tenham a percepção de que não valem mais nada, pois desde sempre, as pessoas com idade avançada foram vistas como aquelas que estão se despedindo da vida. Deduzindo-se, de forma errônea, que por ter se aposentado do seu exercício do trabalho, de suas funções, também se aposentou da vida. Esse preconceito se estende para outros domínios da vida do ser humano, privando os idosos de várias oportunidades de vivenciar o amor, o lazer, a sexualidade e o prazer.

Alguns artigos discutem sobre a interferência da cultura em relação à assexualidade dos idosos, identificando o preconceito e os tabus direcionados a estes indivíduos. A ideia que a sociedade tem acerca da pratica sexual nessa faixa etária ainda decorre do pensamento de que quando a pessoa alcança a fase da velhice deixa de ser sexual, aderindo a assexualidade. A definição da sexualidade limita-se, em sua maioria, ao órgão genital e ao coito. Quando o idoso não tem a presença de um companheiro fixo, isso representa para alguns, o fim das práticas sexuais. A sexualidade não é representada somente pelo ato sexual e o sexo nem sempre significa a penetração, é necessário separar a genitalidade da sexualidade. $\mathrm{Na}$ medida em que o corpo não responde mais ao desejo, as mudanças sexuais se tornam necessárias e ajudam na expressão da sexualidade em idosos. É preciso esclarecer aos idosos que mesmo com a ausência de um parceiro, a busca pelo prazer pode ser obtida por outras formas e que sua identidade sexual não se estabelece pela presença do outro. Os fatores que podem afetar a expressão da sexualidade ou no ato sexual estão relacionados aos aspectos individuais, fisiológicos e sociais, e mesmo com algumas limitações que podem ocorrer durante a velhice, à satisfação sexual ainda pode permanecer. Os problemas de aceitação da sexualidade nessa fase decorrem da ausência de informação ou no conceito de que a sexualidade esteja restrita a genitalidade. Essa concepção existe tanto na sociedade como entre os idosos (ALENCAR et al., 2014).

No item nas mulheres, a perda de satisfação sexual é inevitável após a menopausa, 40\% dos entrevistados revelaram um desconhecimento do processo climatério/ menopausa entre as mulheres. Igualmente quando 50\% afirmam que a maioria das mulheres idosas é fria sexualmente.

Em conformidade com Sousa (2008), a sexualidade entre os idosos é um tema que tem despertado atualmente um grande interesse cientifico. Lembrando que a idade não dessexualiza o indivíduo, o que ocorre são algumas modificações quantitativas relacionadas à resposta sexual, ou seja, constantemente ocorrem transformações na vida sexual ao longo da evolução do indivíduo, porém o desaparecimento só acontece com a 
morte. Até pouco tempo, ainda se pensava que por volta dos 50 anos era inevitável à perda da função sexual, nas mulheres devido à menopausa e nos homens devido às disfunções da ereção masculina. Além disso, a relação sexual perdia o objetivo de procriação e com isso, tinha-se uma justificativa social. Por meio da concepção de Freud, afirmando o prazer como objetivo da sexualidade humana que se liberou a obrigação do resultado pela reprodução. Sendo que esta tese foi confirmada com a recente necessidade do conceito de saúde sexual e com a dissociação progressiva da definição de reprodução, o que evidencia a independência da vida sexual e sua importância para a realização e o bem-estar do ser humano durante toda sua vida.

Ocorreu uma mudança no padrão sexual dos homens idosos devido aos medicamentos disponíveis no mercado para tratamento de disfunção erétil, possibilitando atividade sexual mais intensa. Em relação às mulheres, estudos revelam que a frequência das relações sexuais diminui devido à menopausa, mas elas continuam com atividade sexual e com a dificuldade de negociar o uso do preservativo com seus parceiros (GIRONDI et al., 2012).

Em relação aos itens que abordam o tema masturbação, 60\% dos idosos entrevistados revelaram certo desconhecimento, contradição e quebra de tabu ao afirmarem que a masturbação pode causar confusão mental e demência em idosos, assim como esse ato pode trazer benefícios para a manutenção da resposta sexual do indivíduo.

A definição de saúde reflete o contexto social, econômico, político e cultural. Isto é, saúde não significa a mesma coisa para todos os indivíduos. Decorre da época, do lugar, da classe social. Dependerá dos valores individuais, das concepções cientificas, religiosas, filosóficas. Da mesma forma, pode-se dizer das doenças. O que se considera doença pode variar muito e pelos mesmos motivos anteriores. Podemos exemplificar com a masturbação que em uma época atrás era considerada uma conduta patológica, podendo resultar ao indivíduo uma desnutrição (devido à perda da proteína contida no esperma) e em distúrbios mentais. O tratamento utilizado para a masturbação era dieta, ou operação realizada para impedir relações sexuais (infibulação), pela imobilização do "paciente" por meio de aparelhos elétricos que davam choques quando o pênis era manipulado e até mesmo pela ablação da genitália (SCLIAR, 2007).

\section{Conclusão}

Com base no estudo realizado, evidenciou-se o interesse dos idosos pela sexualidade e como esta é importante para sua qualidade de vida, pois, devido ao perfil que se encontram hoje: idosos, portadores de AIDS, pessoas vistas pela sociedade como um ser "assexuado", esses indivíduos ainda sofrem muitos preconceitos, mesmo com as facilidades tecnológicas atuais, como o uso de medicamentos para disfunção sexual e reposição hormonal. Infelizmente, os próprios profissionais da saúde esquecem de abordar questões sobre esse tema com seus pacientes idosos contribuindo para a exposição desses indivíduos as doenças sexualmente transmissíveis (DST), incluindo a AIDS, e comprovando o preconceito já antigo que a sociedade tem de que idosos não fazem sexo. É necessária uma conscientização e preparo dos profissionais de saúde para que o processo do envelhecimento da sociedade seja acompanhado de maneira holística por meio de campanhas, políticas públicas, pesquisas científicas, consultas com profissionais de saúde, educação em saúde com divulgação nos meios de comunicação, entre outros, possibilitando aos idosos viverem mais e com uma melhor qualidade de vida.

Sobre o estudo, houve uma dificuldade em conseguir a aplicação dos questionários, pois os idosos com o medo do preconceito dos familiares e da sociedade, não revelam que são portadores de AIDS o que repercutiu no número de questionários aplicados. Além disso, a maioria não mora na mesma cidade satélite onde fazem o acompanhamento no Programa de DST, com isso, só concordavam em participar da pesquisa no dia que tinham agendado a consulta com o infectologista, sendo um obstáculo para aplicação e conclusão do estudo. Isto revela o quanto foi difícil conseguir realizar essa pesquisa. Devemos, como profissionais da saúde, insistir em realizar estudos científicos sobre a temática proposta, pois nossa sociedade está envelhecendo e deve ser cuidada de forma integral. A população precisa compreender que a sexualidade é algo natural para qualquer idade e respeitar as necessidades dessa parcela da população.

\section{Referências}

ALENCAR, D. L. de et. al. Fatores que interferem na sexualidade de idosos: uma revisão integrativa. Ciência e Saúde Coletiva, Rio de Janeiro, v. 19, n. 8, p. 3533-3542, ago. 2014. doi: 10.1590/1413-81232014198.12092013. 
ALMEIDA, T.; LOURENÇO, M. L. Envelhecimento, amor e sexualidade: utopia ou realidade. Revista Brasileira de Geriatria e Gerontologia, Rio de Janeiro, v. 10, n. 1, p. 101-113, jan./abr. 2007.

ARAUJO, V. L. B. de et al. Características da AIDS na terceira idade em um hospital de referência do Estado do Ceará, Brasil. Revista Brasileira de Epidemiologia, São Paulo, v. 10, n. 4, p. 544-554, dez. 2007. doi: 10.1590/ S1415-790X2007000400013.

BASTOS, C. C. et al. Importância atribuída ao sexo por idosos do município de Porto Alegre e associação com a auto percepção de saúde e o sentimento de felicidade. Revista Brasileira de Geriatria e Gerontologia, Rio de Janeiro, v. 15, n. 1, p. 87-95, jan./mar. 2012. doi: 10.1590/ S1809-98232012000100010.

BRASIL. Ministério da Saúde. Doenças infecciosas e parasitárias: guia de bolso. 8 . ed. Brasília: Ministério da Saúde, 2010.

CRUZ, G. E. C. P.; RAMOS, L. R. Idosos portadores de HIV e vivendo com AIDS no contexto da capacidade funcional. Acta Paulista de Enfermagem, São Paulo, v. 25, n. 6, p. 981-983, nov./dez. 2012. doi: 10.1590/S010321002012000600024.

GIRONDI, J. B. R. et al. Perfil epidemiológico de idosos brasileiros que morreram por síndrome da imunodeficiência adquirida entre 1996 e 2007. Acta Paulista de Enfermagem, São Paulo, v. 25, n. 2, p. 302-307, mar./abr. 2012. doi: 10.1590/S0103-21002012000200023.

LAROQUE, M. F. et al. Sexualidade do idoso: comportamento para a prevenção de DST/AIDS. Revista Gaúcha de Enfermagem, Porto Alegre, v. 32, n. 4, p. 774-780, dez. 2011. doi: 10.1590/S1983-14472011000400019.

LEITE, M. T.; MOURA, C.; BERLEZI, E. M. Doenças sexualmente transmissíveis e HIV/AIDS na opinião de idosos que participam de grupos de terceira idade. Revista Brasileira de Geriatria e Gerontologia, Rio de Janeiro, v. 10, n. 3, p. 339-354, set./dez. 2007.
LIMA, A. M.; MAIA, J. C. V.; SOUSA, A. B. Perfil epidemiológico da AIDS em idosos no estado do Pará utilizando dados do sistema de informações de saúde do DATASUS. Revista Paraense de Medicina, Belém, v. 27, n. 4, p. 53-58, out./dez. 2013.

MASCHIO, M. B. M. et al. Sexualidade na terceira idade: medidas de prevenção para doenças sexualmente transmissíveis e AIDS. Revista Gaúcha de Enfermagem, Porto Alegre, v. 32, n. 3, p. 583-589, set. 2011. doi: 10.1590/ S1983-14472011000300021.

MACHIESQUI, S. R. et al. Pessoas acima de 50 anos com AIDS: implicações para o dia-a-dia. Escola Anna Nery, Rio de Janeiro, v. 14, n. 4, p. 726-731, dez. 2010. doi: $10.1590 /$ S1414-81452010000400011.

MORAES, K. M. et al. Companheirismo e sexualidade de casais na melhor idade: cuidando do casal idoso. Revista Brasileira de Geriatria e Gerontologia, Rio de Janeiro, v. 14, n. 4, p. 787-798, out./dez. 2011. doi: 10.1590/S180998232011000400018 .

OKUNO, M. F. P. et al. Conhecimento e atitudes sobre sexualidade em idosos portadores de HIV/AIDS. Acta Paulista de Enfermagem, São Paulo, v. 25, n. 1, p. 115-121, jan./fev. 2012. doi: 10.1590/S0103-21002012000800018.

SCLIAR, M. Historia do conceito de saúde. Physis, Rio de Janeiro, v. 17, n. 1, p. 29-41, abr. 2007. doi: 10.1590/ S0103-73312007000100003.

SOUSA, J. L. Sexualidade na terceira idade: uma discussão da AIDS, envelhecimento e medicamentos para disfunção erétil. Jornal Brasileiro de Doenças Sexualmente Transmissíveis, Niterói, v. 20, n. 1, p. 59-64, jan./mar. 2008.

VIANA, H. B.; GUIRARDELLO, E. B.; MADRUGA, V. A. Tradução e adaptação cultural da escala ASKAS: Aging Sexual Knowledge and Attitudes Scale em idosos brasileiros. Texto e Contexto: Enfermagem, Florianópolis, v. 19 , n. 2, p. 238-245, jun. 2010. doi: 10.1590/S010407072010000200004 . 